\title{
Bound and unbound nuclear systems at the drip lines: a one-dimensional model
}

\author{
L Moschini $^{1}{ }^{2}{ }^{3}$, F Pérez-Bernal ${ }^{4}$ and A Vitturi ${ }^{12}$ \\ ${ }^{1}$ Dipartimento di Fisica e Astronomia "G. Galilei", Padova, Italy \\ ${ }^{2}$ INFN, Sezione di Padova, Padova, Italy \\ ${ }^{3}$ Departamento de Física Atómica, Molecular y Nuclear, Universidad de Sevilla, \\ Apartado 1065, E-41080 Sevilla, Spain \\ ${ }^{4}$ Departamento de Física Aplicada, Unidad Asociada al IEM-CSIC, Universidad de \\ Huelva, E-21071 Huelva, Spain \\ E-mail: laura.moschini@pd.infn.it
}

\begin{abstract}
We construct a one-dimensional toy model to describe the main features of Borromean nuclei at the continuum threshold. The model consists of a core and two valence neutrons, unbound in the mean potential, that are bound by a residual point contact density-dependent interaction. Different discretization procedures are used (Harmonic Oscillator and Transformed Harmonic Oscillator bases, or use of large rigid wall box). Resulting energies and wave functions, as well as inelastic transition intensities, are compared within the different discretization techniques, as well as with the exact results in the case of one particle and with the results of the dineutron cluster model in the two particles case. Despite its simplicity, this model includes the main physical features of the structure of Borromean nuclei in an intuitive and computationally affordable framework, and will be extended to direct reaction calculations.
\end{abstract}

\section{Introduction}

One of the most relevant research lines in Nuclear Structure nowadays is the investigation, both experimentally and theoretically, of nuclei under extreme conditions and, in particular, nuclei far from the stability line. Examples of such systems are nuclei with large neutron excess, with the barely bound outermost ones creating an extended density distribution, named as halo. Nuclei that do not accept more neutrons mark on the isotope chart the neutron drip line, and along this line truly enticing and striking novel nuclear structure phenomena are being observed. Nowadays measuring the properties of such nuclei is the goal of the main experimental nuclear facilities around the world $[1,2]$.

The theoretical description of halo nuclei is strongly characterized by its weaklybound nature. Bound nuclei in the vicinities of the stability valley can be modeled within a mean field potential partially filled with nucleons (protons and neutrons). In this simplified model the stable ground-state configuration is schematically depicted in 
figure 1a, while the lowest nuclear excitations in this picture can be obtained by promoting one or more nucleons to the still bound higher energy states, as shown in figure 1b. How is this simplified picture affected as nuclei get close to the neutron drip line? In this case the neutron Fermi energy approaches to zero, as shown in figure 1c and the corresponding neutron excited states must involve the promotion of a neutron to continuum states (figure 1d). The last nucleons are so weakly-bound that the addition of any correlation to the simple mean field model inevitably involves the inclusion of the continuum in the system description.

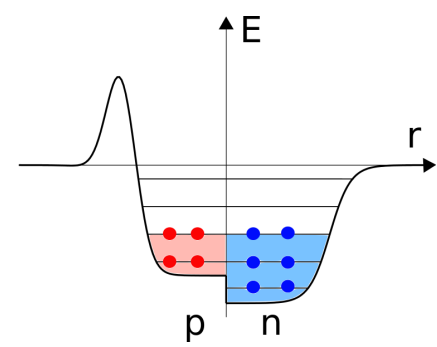

(a)

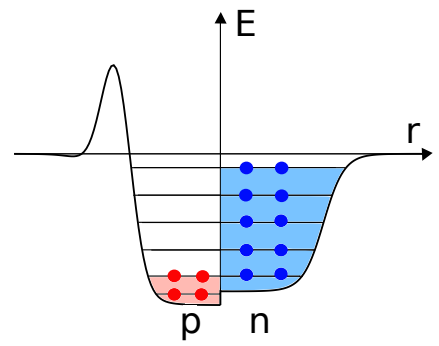

(c)

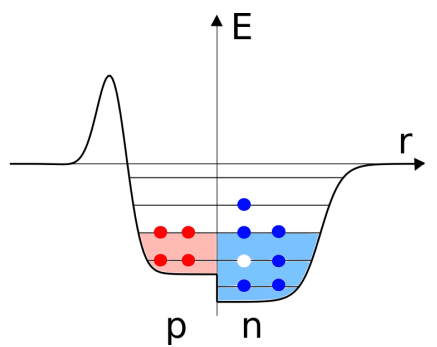

(b)

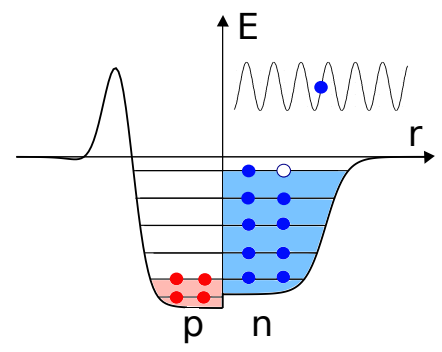

(d)

Figure 1: Schematic single-particle configuration of the ground-state (a) and its particlehole excitation (b) for a nucleus close to the stability valley. Corresponding situation for a system close to the neutron drip line, with ground-state (c) and its excitation of a nucleon to the continuum (d).

In accordance with this picture we present in this memory results for a onedimensional (1D) toy model of a particular kind of halo nuclei: the so-called Borromean two-neutron halo nuclei. Borromean systems are three-body systems (core plus two particles) that are bound systems with no bound state in the possible binary subsystems. The seminal best known examples of Borromean nuclei are ${ }^{6} \mathrm{He}$ and ${ }^{11} \mathrm{Li}$, but other Borromean nuclear systems have been proposed, e.g. ${ }^{14} \mathrm{Be}$ and ${ }^{22} \mathrm{C}[3]$.

The main ingredients of the present systems are a mean field 1D Woods-Saxon potential, with all bound levels supposed to be totally filled and inert, plus two extra neutrons added to this core. The resulting system, unbound at the mean-field level, is bound due the action of a residual point contact density-dependent interaction. It is clear that the description of such a system requires an adequate consideration of the 
role of the continuum. In order to do so we first deal with the one body mean field Woods-Saxon potential in section 2. We select a set of Woods-Saxon potential parameter values that qualitatively model a nuclear structure problem and discretize the system continuum, using a finite set of normalizable (square-integrable) pseudostates; three different approaches have been followed. The first one consists in diagonalizing the Woods-Saxon potential Hamiltonian matrix in a truncated Harmonic Oscillator (HO) basis, the second makes use of a local scale transformation to construct a truncated Transformed Harmonic Oscillator (THO) basis, while the third one uses a rigid wall box (BOX) to achieve continuum discretization. We present in section 2.2 and 2.3 results obtained for eigenvalues, eigenfunctions, and other quantities of interest (single-particle transition of $x$ and $x^{2}$ operators, total strength, and sum rules), studying and comparing the convergence properties of the different approaches. Since the one-body case can be directly solved without resorting to continuum discretization, our results can be also compared to the "exact" values.

Once the pseudostate description of the model Woods-Saxon potential has been set up, we proceed in section 3 to state the two-body problem (two neutrons plus WoodsSaxon core) in 1D, to define an appropriate basis, and to construct bound and excited states by diagonalizing the system Hamiltonian with the different continuum discretization procedures described in section 2. Besides the discussion on the convergence of eigenvalues and eigenfunctions (section 3.2), we compute other quantities of interest as transition intensities of $x$ and $x^{2}$ operators. The obtained results are compared in section 3.3 with those obtained describing the same system (core plus two neutrons) within the "popular" di-neutron cluster model. The last section contains some concluding remarks and suggestions of future investigations along the present line of research.

Though the simplification achieved devising our 1D model precludes us from comparing directly to real nuclei, we expect that the present toy model, notwithstanding its simplicity, contains the basic physical ingredients for a correct description of the problem under study. A solution of a simplified toy model frequently casts light upon a physical problem whose full solution is hindered by mathematical complexities or is plainly not possible. Reduced dimensionality models of application in Nuclear Physics can be found e. g. in $[4,5,6]$. In particular, some results for the model investigated in the present memory can already be found in [7]. As a last remark we would like to mention that the calculations presented have been carried out using FORTRAN90, GNU-Octave, and PERL codes developed for this purpose [8], and that part of the results were presented by one of the authors (LM) for the obtention of her master's degree [9].

\section{One-body problem}

In many-body systems at the drip lines, e. g. halo nuclei, it is mandatory to include continuum effects in the system description, starting with a single-particle basis that 
must include the positive energy part of the spectrum. In this section we start therefore presenting different pseudostate methods to model weakly-bound one-body quantum systems.

In pseudostate methods continuum wave functions are obtained as eigenstates of the system Hamiltonian matrix in a truncated basis of square-integrable wave functions. Continuum discretization methods include for example the use of Harmonic Oscillator and Transformed Harmonic Oscillator basis, the use of large rigid wall box or, as extensively used in reaction theories, the average method that build a basis of normalized bin functions superposing true continuum wave functions. The results of the different methods are tested in the case of a 1D Woods-Saxon potential with parameters chosen in order to mimic the nuclear case for a light system.

\subsection{Continuum Discretization with Pseudostates}

The problem of particles moving in a one dimensional Woods-Saxon mean field can be solved diagonalizing the Hamiltonian in different bases. In fact the solution of the 1D time-independent Schroedinger equation (TISE)

$$
\hat{H}_{1 b} \psi(x)=E \psi(x) \rightarrow\left[-\frac{\hbar^{2}}{2 \mu} \frac{d^{2}}{d x^{2}}+V_{W S}(x)\right] \psi(x)=E \psi(x),
$$

is reduced to the problem of building and diagonalizing a Hamiltonian matrix within each of the considered bases. This will provide the eigenfunctions and the corresponding energies of the problem, that are not limited to the bound states, but also pseudostates in the continuum (positive energy states). The 1D Woods-Saxon potential is

$$
V_{W S}(x)=\frac{V_{0}}{1+e^{\frac{|x|-R}{\alpha}}}
$$

and the chosen potential parameters are $V_{0}=-50.00 \mathrm{MeV}, R=2.00 \mathrm{fm}, \alpha=0.40$ $\mathrm{fm}$, and $\mu=0.975 \mathrm{amu}$. This particular set of parameters has been chosen to set up a 1D toy model representative of a light-mass weakly-bound nucleus. With this choice of parameters the system has in fact three single-particle bound states whose energies are presented in figure 2, with a weakly-bound third energy level (-0.51 MeV binding energy).

The most popular approach in Quantum Mechanics to construct a basis of pseudostates is provided by the Harmonic Oscillator [11]. To solve the TISE in a 1D $\mathrm{HO}$ basis, the starting point is to generate a truncated $N$ dimensional basis set of $1 \mathrm{D}$ $\mathrm{HO}$ wave functions

$$
\phi_{i}^{H O}(x)=M_{i} \sqrt{a} H_{i}(a x) e^{-a^{2} x^{2} / 2} ; \quad i=0, \ldots, N-1 .
$$

The parameter $a=\sqrt[4]{\mu K / \hbar^{2}}$ is the inverse of the oscillator length with $K$ equal to the 4-th constant of the Harmonic Oscillator, $H_{i}(a x)$ is the $i$-th Hermite polynomial, and 


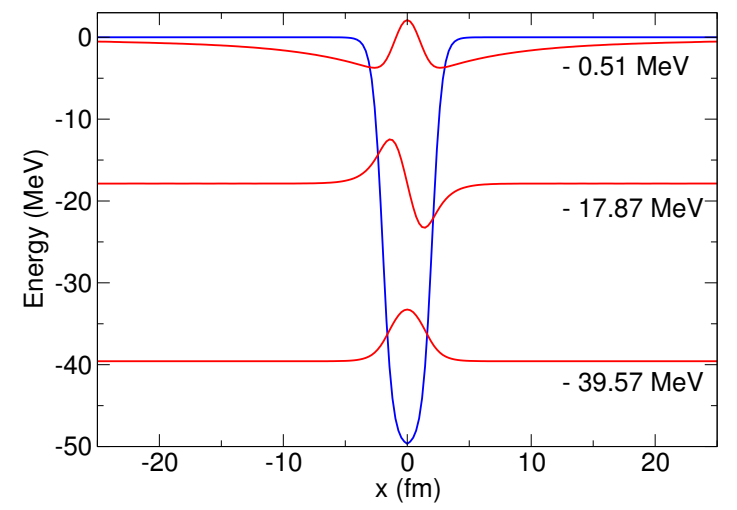

Figure 2: The model Woods-Saxon 1D potential and its bound wave functions. Those shown in the figure have been computed using HO basis with $\mathrm{N}=50$.

$M_{i}$ is a normalization constant

$$
M_{i}=\frac{1}{\sqrt{2^{i} i ! \sqrt{\pi}}} .
$$

The HO basis can be easily constructed and the necessary integral calculations are simplified making use of the Hermite polynomial recurrence relation [12]. Once the matrix is diagonalized, we obtain a set of one-body eigenvalues, $E_{i}^{H O}$, and eigenfunctions, $\psi_{i}^{H O}(x)$. The eigenstates are linear combinations of the basis elements

$$
\psi_{i}^{H O}(x)=\sum_{k=0}^{N-1} \alpha_{i k}^{H O} \phi_{k}^{H O}(x) ; i=0, \ldots, N-1 .
$$

A disadvantage of the $\mathrm{HO}$ basis when used as a basis in variational methods to model bound states is its Gaussian asymptotic behavior, compared to the true bound states exponential behavior. This is even more so in continuum pseudostates. This fact explains the success of the THO basis. A THO basis consists of a HO basis to which a local scale transformation (LST) $s(x)$ has been applied. The aim of this transformation is to alter the HO wave functions asymptotic behavior. For details see, e. g. [13, 14, 15].

To solve the problem in a $1 \mathrm{D}$ THO basis our starting point is a truncated $N$ dimensional basis set $\phi_{i}^{H O}(x)$ of 1D HO wave functions, which has to be scaled into the new basis as follows

$$
\phi_{i}^{T H O}(x)=\sqrt{\frac{d s(x)}{d x}} \phi_{i}^{H O}(s(x)) ; \quad i=0, \ldots, N-1,
$$

according to the analytical LST function (see $[15,16]$ )

$$
s(x)=\left[x^{-m}+(\gamma \sqrt{x})^{-m}\right]^{-\frac{1}{m}}
$$

that is valid for $x>0$; for negative $x$ values we impose that $s(x)$ is an odd function: $s(-x)=-s(x)$. The quantity $\gamma$ is a parameter of the LST and is a variable parameter of the calculation. Once the TISE for the 1D mean field Woods-Saxon potential (2) is solved using the basis (6), we obtain a set of discrete eigenvalues, $E_{i}^{T H O}$, and 
eigenfunctions, $\psi_{i}^{T H O}(x)$, and the latter can be written as a linear combination of the THO basis states

$$
\psi_{i}^{T H O}(x)=\sum_{k=0}^{N-1} \alpha_{i k}^{T H O} \phi_{k}^{T H O}(x) ; \quad i=0, \ldots, N-1 .
$$

A third way to obtain a continuum discretization is making use of a rigid box of radius $x_{b}$, with $x_{b}$ being large enough compared to the potential range

$$
V_{b}(x)=\left\{\begin{array}{cl}
V_{W S} & \text { if }|x|<x_{b}, \\
\infty & \text { if }|x| \geq x_{b} .
\end{array}\right.
$$

This potential-in-a-box problem can be solved using standard numerical techniques for the solution of differential equations, as for example the Numerov approach. Once the 1D TISE for the mean field Woods-Saxon potential (2) is solved, we obtain the eigenenergies $E_{i}^{B O X}$ and the one-body eigenfunctions $\psi_{i}^{B O X}(x)$.

\subsection{Model Woods-Saxon Potential Energies and Wavefunctions}

As previously stated, the PS approach that we follow implies the solution of the model hamiltonian with different procedures. The obtained results depend on the basis dimension for $\mathrm{HO}$ and THO methods or on the box radius $x_{b}$ for the BOX method. We proceed to study the convergence of eigenvalues and eigenfunctions when these parameters are varied. We focus not only on the energies, but also on the wave functions asymptotic behavior. A proper description of the wave functions tails is, in fact, crucial to reasonably describe the reaction properties of our system. This is even more important when, as in our chosen specific case, the Woods-Saxon potential has a very weakly-bound state $\left(E_{2}=-0.51 \mathrm{MeV}\right)$, as shown in figure 3 .

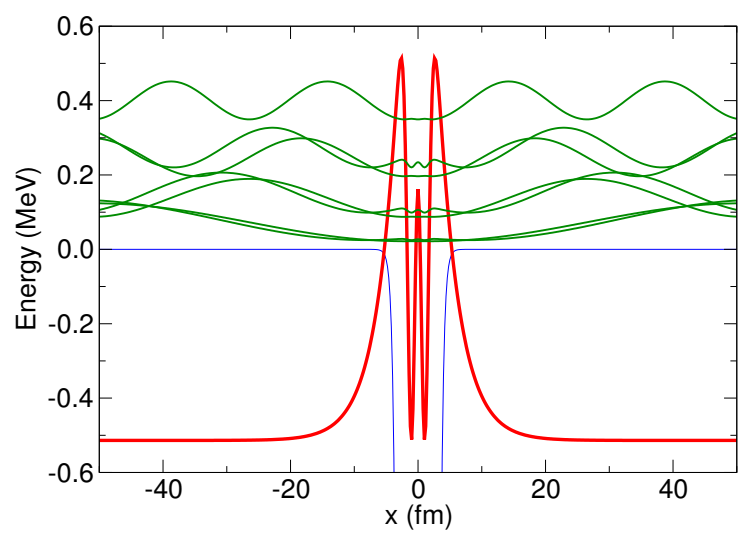

Figure 3: The Woods-Saxon model potential (thin blue), last squared bound wave function (thick red) and the first squared continuum PSs (green) obtained with the Hamiltonian matrix diagonalization (in particular BOX with $x_{b}=100 \mathrm{fm}$ ).

In the $\mathrm{HO}$ case the key parameter is the basis dimension $\mathrm{N}$; so we check the results convergence with a varying basis size. In addition to the value of $\mathrm{N}$, a second model 
parameter is the inverse oscillator length $a$. The inverse oscillator length determines the curvature of the $\mathrm{HO}$ potential at the origin and thus how wide the potential is (see Appendix A).

In the THO case results depend mainly on the basis dimension $\mathrm{N}$ and the parameter ratio $\gamma / b$, where $b=1 / a$ is the oscillator length. The second LST parameter, m, affects very lightly to the calculations and has been fixed to a constant $\mathrm{m}=4$ value following [14]. The ratio $\gamma / b=\left(\frac{8 \mu \varepsilon}{\hbar^{2}}\right)^{1 / 4}$ gives an extra degree of freedom to the approach compared to the HO case. As discussed in $[14,15]$, the value of $\frac{\gamma^{2}}{2 b^{2}}$ can be considered as an effective momentum value, $k_{e f f}$, and the asymptotic value of the basis functions is $e^{-\frac{\gamma^{2}}{2 b^{2}}|x|}$. As $\gamma / b$ increases (decreases) the basis spatial extension decreases (increases). Therefore, for small $\gamma / b$ values the positive eigenvalues tend to concentrate at lower energies, and the $\gamma / b$ ratio controls the density of PSs as a function of the excitation energy. This useful property of THO basis makes this approach an interesting alternative to HO [15]. For large $\gamma / b$ values the THO reaches the HO limit. The improved asymptotic wavefunction behavior is ascertained computing the bound states energy convergence. If we fix the value of $5 \mathrm{keV}$ as the energy convergence goal, an $N=50$ basis is needed for $\gamma / b=2.4 \mathrm{fm}^{-1 / 2}$ (as in the HO case) while it is enough with $N=20$ for $\gamma / b=1.2 \mathrm{fm}^{-1 / 2}$. All THO results have been calculated for $\gamma / b=1.2 \mathrm{fm}^{-1 / 2}$.

The third option considered for obtaining pseudostates is enclosing the potential in a rigid wall box. The main parameter when solving the problem of a potential in a rigid wall box is $x_{b}$, the box width.

In the left panels of figure 4 we depict the eigenvalues of the model Woods-Saxon potential as a function of the basis dimension $\mathrm{N}$ or the box radius $x_{b}$, depending on the method. Negative energy levels converge to the bound state energies quite fast. We consider that an energy level is converged when $\Delta E \leq 5 \mathrm{keV}$ for a dimension increment $\Delta N=10$. As expected, the convergence is much faster for the ground and first excited states than for the weakly-bound second excited state.

In the right panels of figure 4 we show for different parameter values the weaklybound state wave function tails, where the major differences can be found. Large N values (in $\mathrm{HO}$ and THO cases) are required to extend towards large $x$ values, reproducing the exponential behaviour. Note that the THO approach leads to a faster convergence than the bare HO approach. For the BOX case we show the wave function tail for different box sizes.

\subsection{Excitation Matrix Elements and Sum Rules}

In this subsection we present results for the transition probabilities for the $x$ and $x^{2}$ operator from the weakly-bound state to excited states at positive energies. These are the $1 \mathrm{D}$ equivalent of the dipole and quadrupole transition strengths to the continuum 

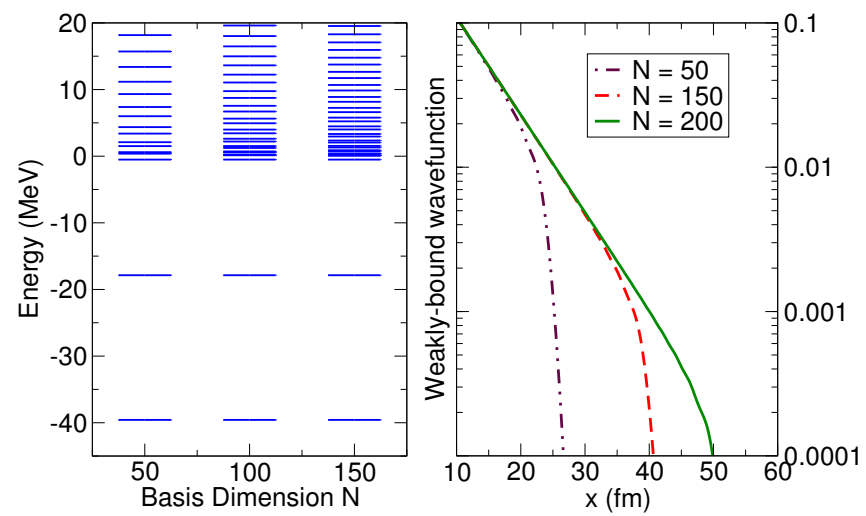

(a) $\mathrm{HO}$

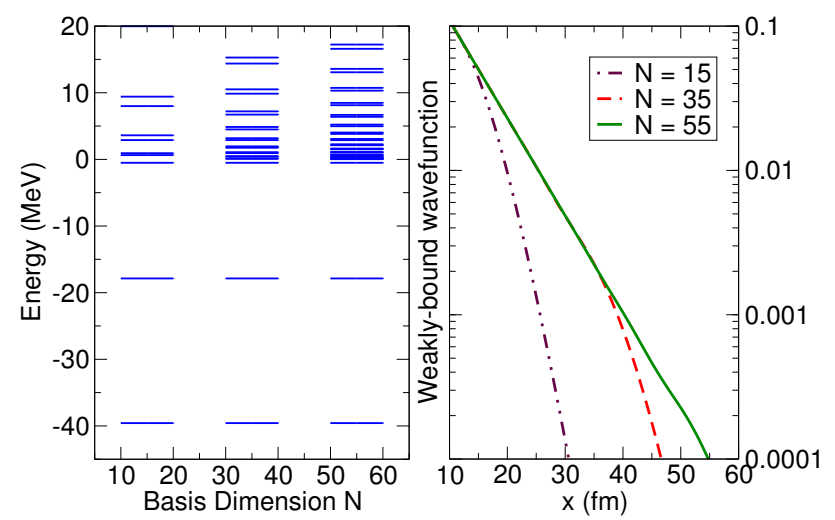

(b) $\mathrm{THO}$

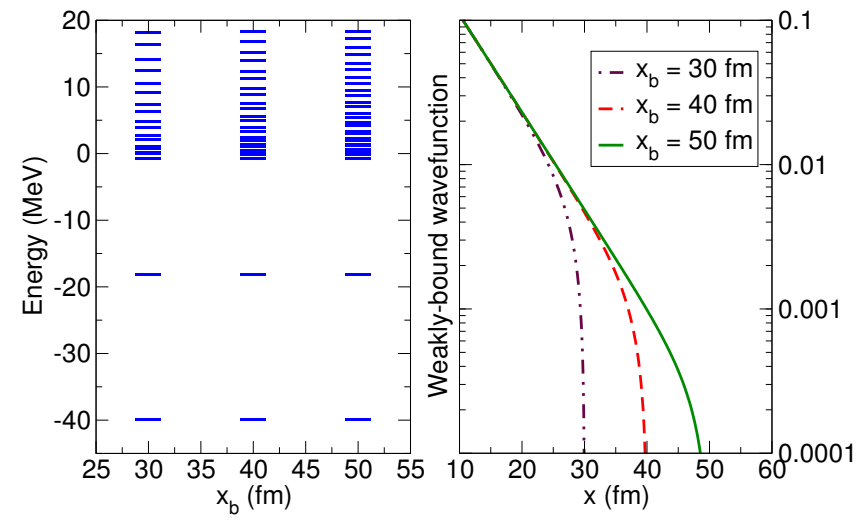

(c) BOX

Figure 4: Left panels: Eigenvalues of the Woods-Saxon model potential as a function of the number of basis states (in the $\mathrm{HO}$ and THO case) and as a function of the box radius (in the BOX case). Right panels: asymptotic spatial dependence of the weakly-bound state wave function (in logarithmic scale) as a function of $x$ for different parameters values. 
in 3D. As it is well known, the low binding energy of weakly-bound systems strongly affects the response to the continuum and, therefore, the convergence of these matrix elements is a crucial test for the discretization procedures.

The transition probability between bound states or between a bound state and a continuum pseudostate (see the discussion in [17]) can be written as

$$
B_{n}(b \rightarrow i)=\left|\left\langle\Psi_{b}\left|\mathcal{O}_{n}(x)\right| \Psi_{i}\right\rangle\right|^{2} .
$$

with $\mathcal{O}_{n}(x)=x$ and $x^{2}$ for $\mathrm{n}=1$ and 2 , respectively. Therefore we need to compute the integrals

$$
\left\langle\Psi_{b}\left|\mathcal{O}_{n}(x)\right| \Psi_{i}\right\rangle=\int_{-\infty}^{+\infty} d x \Psi_{b}^{*}(x) \mathcal{O}_{n}(x) \Psi_{i}(x)
$$

By changing the $\mathrm{N}$ or $x_{b}$ parameters we expect a difference in the density of continuum levels (as previously shown in the left panels of figure 4). For example, in figure 5 we present the different distributions of $B_{1}$ intensities obtained with the THO procedure for $\mathrm{N}=35$ and $\mathrm{N}=85$, where this effect can be easily appreciated.

The $\mathrm{B}_{1}$ and $\mathrm{B}_{2}$ distributions for $\mathrm{HO}$, THO, and $\mathrm{BOX}$ cases are shown in figure 6 for the calculations starting from the weakly-bound third state $\Psi_{2}(x)$. For a better comparison of the convergence properties, in the last row of panels of figure 6 we also display the summed $\operatorname{Tot}\left(B_{1}\right)_{2 \rightarrow i}=\sum_{i=1}^{N}\left|\left\langle\Psi_{2}|x| \Psi_{i}\right\rangle\right|^{2}$ and $\operatorname{Tot}\left(B_{2}\right)_{2 \rightarrow i}=\sum_{i=1}^{N}\left|\left\langle\Psi_{2}\left|x^{2}\right| \Psi_{i}\right\rangle\right|^{2}$ transition intensities as function of the energy. These should be calculated including in the $\left\{\Psi_{i}\right\}$ set only those states above the initial state $\Psi_{2}$, i.e. only the continuum states in our case. However, in the calculation of the summed strength we have included also the transition to lower energy bound states, which in principle are occupied by the core particles and thus Pauli forbidden. In this way we can in fact compare the results with the sum rules, that encompass all the states.

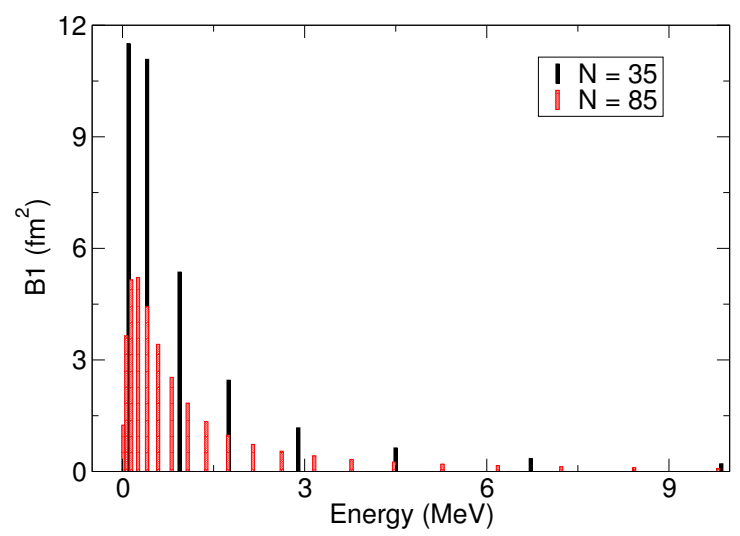

Figure 5: $\mathrm{B}_{1}$ transition intensities calculated for the $b=2$ weakly-bound state in THO case with $\mathrm{N}=35$ (dark bars) and $\mathrm{N}=85$ (red light bars).

For our one-body problem, the Total Strength Sum Rule (TSSR) for an operator $\mathcal{O}$ is

$$
\mathcal{S}_{T}^{(b)}(\mathcal{O}, N)=\sum_{i=0}^{N-1}\left|\left\langle\Psi_{b}|\mathcal{O}(x)| \Psi_{i}\right\rangle\right|^{2}
$$




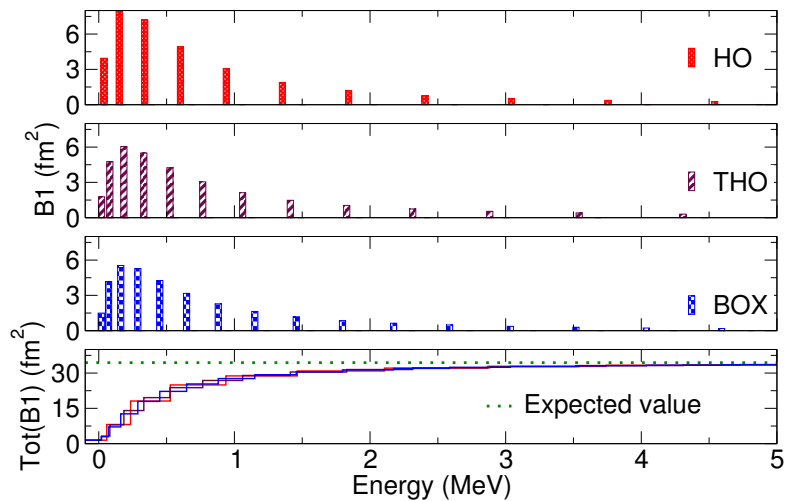

(a) B1

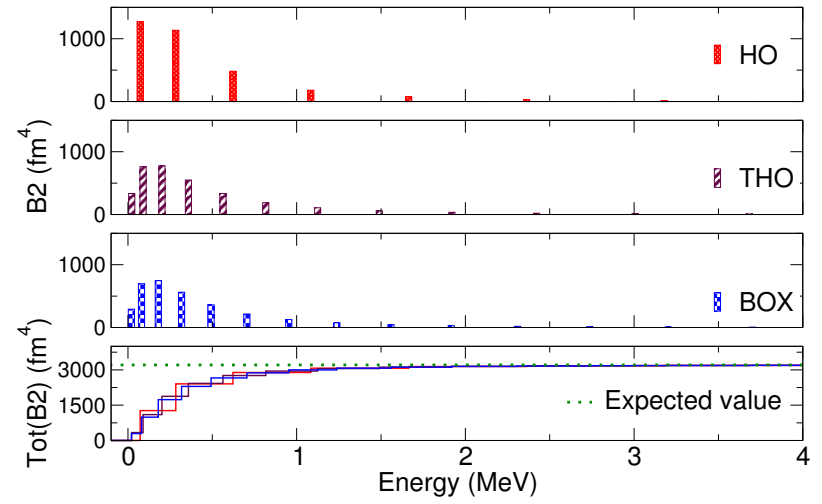

(b) B2

Figure 6: $\mathrm{B}_{1}$ (upper four panels) and $\mathrm{B}_{2}$ (lower four panels) energy distributions calculated starting from the third bound state in HO, THO, and BOX cases (using $\mathrm{N}_{H O}=250, \mathrm{~N}_{T H O}=85$, and $\left.x_{b}=100 \mathrm{fm}\right)$. In the last rows the $\operatorname{Tot}\left(B_{1}\right)$ and $\operatorname{Tot}\left(B_{2}\right)$ convergence to the expected values (green dotted lines) are reported as a function of energy.

where $\left|\Psi_{b}\right\rangle$ is the bound state wave function $\left(\left|\Psi_{2}\right\rangle\right.$ in our case) and $\left\{\left|\Psi_{i}\right\rangle\right\}_{i=1}^{N}$ is the set of bound states plus pseudostates. In the large $\mathrm{N}$ limit, due to the basis completeness, the TSSR is given by

$$
\lim _{N \rightarrow \infty} \mathcal{S}_{T}^{(b)}(\mathcal{O}, N)=\left\langle\Psi_{b}\left|\mathcal{O}^{2}(x)\right| \Psi_{b}\right\rangle
$$

and can be easily calculated numerically from the bound state wave functions. These values are depicted in figure 6 as green horizontal dotted lines and, as one can see, we find a good agreement for all discretization procedures.

Another useful quantity to assess the goodness of a continuum discretization is the Energy Weighted Sum Rule (EWSR)

$$
\mathcal{E}_{W}^{(b)}(\mathcal{O}, N)=\sum_{i=0}^{N-1}\left(E_{i}-E_{b}\right)\left|\left\langle\Psi_{b}|\mathcal{O}(x)| \Psi_{i}\right\rangle\right|^{2},
$$

where, again, $\left|\Psi_{b}\right\rangle$ is the bound state wave function and $\left\{\left|\Psi_{i}\right\rangle\right\}_{i=1}^{N}$ is the set of bound 
states plus pseudostates. In the large $\mathrm{N}$ limit the EWSR is given by

$$
\lim _{N \rightarrow \infty} \mathcal{E}_{W}^{(b)}\left(\mathcal{O}_{n}, N\right)=\frac{1}{2} \frac{\hbar^{2}}{\mu}\left\langle\Psi_{b}\left|\left(\frac{d \mathcal{O}_{n}(x)}{d x}\right)^{2}\right| \Psi_{b}\right\rangle .
$$

Thus in the case of $\mathcal{O}_{1}=x$, it results

$$
\mathcal{E}_{W}^{(b)}(x, N \rightarrow \infty)=\frac{1}{2} \frac{\hbar^{2}}{\mu}
$$

while for $\mathcal{O}_{2}=x^{2}$

$$
\mathcal{E}_{W}^{(b)}\left(x^{2}, N \rightarrow \infty\right)=2 \frac{\hbar^{2}}{\mu}\left\langle\Psi_{b}\left|x^{2}\right| \Psi_{b}\right\rangle
$$

These values are compared in table 1 with those calculated for different values of $\mathrm{N}$ for the $\mathrm{HO}$ and THO discretization procedures and in table 2 for different values of $x_{b}$ for the BOX procedure, summing the contributions of the different pseudostates. As one can see from the convergence of the different sum rules, smaller $\mathrm{N}$ values are required in the THO than in the HO case. In the BOX case a rather large value of $x_{b}$ is necessary.

\begin{tabular}{lcc|lcc}
$\mathrm{N}$ & $\begin{array}{c}\text { TO } \\
\text { TSSR } \\
\left(\mathrm{fm}^{2}\right)\end{array}$ & $\begin{array}{c}\text { EWSR } \\
\left(\frac{\hbar^{2}}{\mu}\right)\end{array}$ & $\mathrm{N}$ & $\begin{array}{c}\text { THO } \\
\text { TSSR } \\
\left(\mathrm{fm}^{2}\right)\end{array}$ & $\begin{array}{c}\text { EWSR } \\
\left(\frac{\hbar^{2}}{\mu}\right)\end{array}$ \\
\hline 15 & 16.26 & 0.500 & 8 & 15.34 & 0.508 \\
100 & 34.38 & 0.500 & 20 & 33.43 & 0.500 \\
150 & 34.44 & 0.499 & 35 & 34.42 & 0.500 \\
200 & 34.44 & 0.500 & 55 & 34.44 & 0.500 \\
250 & 34.44 & 0.500 & 85 & 34.44 & 0.500 \\
\hline & $\mathbf{3 4 . 4 4}$ & $\mathbf{0 . 5 0 0}$ & & $\mathbf{3 4 . 4 4}$ & $\mathbf{0 . 5 0 0}$
\end{tabular}

(a) $\mathrm{B}_{1}$

\begin{tabular}{lcc|lcc}
$\mathrm{N}$ & $\begin{array}{c}\mathrm{HO} \\
\text { TSSR } \\
\left(\mathrm{fm}^{4}\right)\end{array}$ & $\begin{array}{c}\text { EWSR } \\
\left(\frac{\hbar^{2}}{\mu}\right)\end{array}$ & $\mathrm{N}$ & $\begin{array}{c}\text { THO } \\
\text { TSSR } \\
\left(\mathrm{fm}^{4}\right)\end{array}$ & $\begin{array}{c}\text { EWSR } \\
\left(\frac{\hbar^{2}}{\mu}\right)\end{array}$ \\
\hline 15 & 242.7 & 31.59 & 8 & 200.3 & -1.592 \\
100 & 3137 & 68.80 & 20 & 2576 & 72.11 \\
150 & 3205 & 68.89 & 35 & 3187 & 68.85 \\
200 & 3212 & 68.90 & 55 & 3212 & 68.95 \\
250 & 3213 & 68.91 & 85 & 3212 & 68.91 \\
\hline & $\mathbf{3 2 1 3}$ & $\mathbf{6 8 . 9 1}$ & & $\mathbf{3 2 1 3}$ & $\mathbf{6 8 . 9 1}$
\end{tabular}

(b) $\mathrm{B}_{2}$

Table 1: The $\mathrm{B}_{1}(\mathcal{O}=x)$ (a) and $\mathrm{B}_{2}\left(\mathcal{O}=x^{2}\right)$ (b) "exact" sum rules values in bold are compared with those calculated for different values of $\mathrm{N}$ for the $\mathrm{HO}$ and $\mathrm{THO}$ bases summing the contributions of the different pseudostates. 


\begin{tabular}{|c|c|c|c|c|}
\hline $\begin{array}{l}x_{b} \\
(\mathrm{fm})\end{array}$ & $\begin{array}{c}\mathrm{B} 1 \\
\mathrm{TSSR} \\
\left(\mathrm{fm}^{2}\right)\end{array}$ & $\begin{array}{c}\text { EWSR } \\
\left(\frac{\hbar^{2}}{\mu}\right)\end{array}$ & $\begin{array}{l}\mathrm{B} 2 \\
\mathrm{TSSR} \\
\left(\mathrm{fm}^{4}\right) \\
\end{array}$ & $\begin{array}{c}\text { EWSR } \\
\left(\frac{\hbar^{2}}{\mu}\right)\end{array}$ \\
\hline 15.00 & 23.61 & 0.499 & 733.3 & 67.23 \\
\hline 35.00 & 34.18 & 0.500 & 2990 & 68.37 \\
\hline 55.00 & 34.45 & 0.500 & 3211 & 68.91 \\
\hline 85.00 & 34.45 & 0.499 & 3214 & 68.90 \\
\hline \multirow[t]{2}{*}{100.0} & 34.44 & 0.499 & 3213 & 68.90 \\
\hline & 34.44 & 0.500 & 3213 & 68.91 \\
\hline
\end{tabular}

Table 2: The $\mathrm{B}_{1}(\mathcal{O}=x)$ (left) and $\mathrm{B}_{2}\left(\mathcal{O}=x^{2}\right)$ (right) "exact" sum rules values in bold are compared with those calculated for different values of $x_{b}$ for the BOX procedure, summing the contributions of the different pseudostates.

As it is apparent from figure 6 the distribution of transition matrix elements to continuum states follows the expected threshold shape of the multipole strength for weakly-bound systems.

To emphasize this point it is interesting to compare the result obtained using the discretized pseudostates with an analytic expression. This formula is obtained by approximating the weakly-bound wave function by an exponential and the contiuum state by a free plane wave. In this case, in fact, aside from a normalization factor and as a function of the momentum $k$, the result is

$$
\frac{d B_{1}}{d k} \propto\left|\int_{-\infty}^{+\infty} d x e^{-k_{b}|x|} x e^{i k x}\right|^{2} \propto \frac{k_{b}^{2} k^{2}}{\left(k^{2}+k_{b}^{2}\right)^{4}}
$$

where $k$ is the momentum in the continuum and $k_{b}=\sqrt{\frac{2 \mu\left|E_{b}\right|}{\hbar^{2}}}$ is the momentum associated to the weakly-bound second state.

It is also possible for the one-body case to provide a comparison with an "exact" calculation. In $1 \mathrm{D}$ for each positive energy there are two degenerate continuum wave functions with momentum $k= \pm \sqrt{\frac{2 \mu E}{\hbar^{2}}}$, one incoming from the left and the other from the right. For each energy one could take the symmetric and antysimmetric combinations of the momentum normalized continuum wave functions

$$
\begin{aligned}
& \Psi_{ \pm k}^{s}=\frac{1}{\sqrt{2}}\left[\Psi_{+k}(x)+\Psi_{-k}(x)\right], \\
& \Psi_{ \pm k}^{a}=\frac{1}{\sqrt{2}}\left[\Psi_{+k}(x)-\Psi_{-k}(x)\right], \\
& \left\langle\Psi_{k}^{\rho} \mid \Psi_{k^{\prime}}^{\rho^{\prime}}\right\rangle=\delta_{\rho \rho^{\prime}} \delta\left(k-k^{\prime}\right),
\end{aligned}
$$

where $\rho=s, a$. Since our weakly-bound state is symmetric, only antisymmetric states are connected by the $x$ operator. So the $B_{1}$ distribution for the "exact" case is given by

$$
\frac{d B_{1}}{d k} \propto\left|\int_{-\infty}^{+\infty} d x \Psi_{b}^{*}(x) x \Psi_{ \pm k}^{a}(x)\right|^{2} .
$$


The comparison (in the BOX case) between analytical, "exact", and pseudostates results for $B_{1}$ is shown in figure (7). The alternative methods are in good agreement.

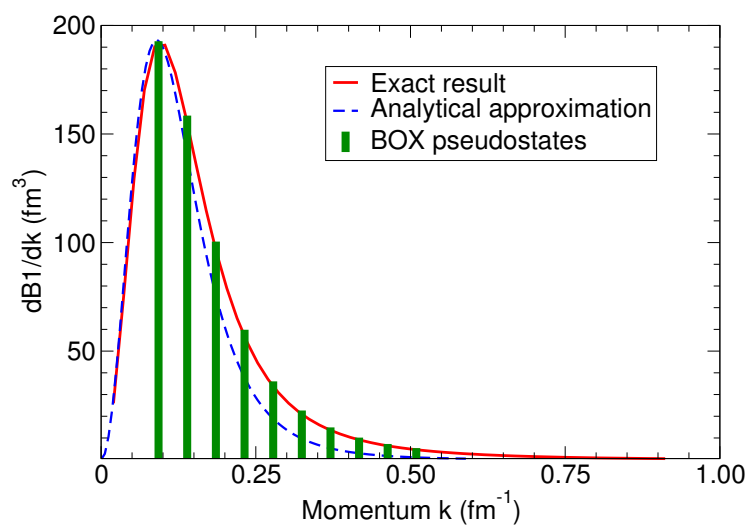

Figure 7: $\frac{d B_{1}}{d k}$, as a function of momentum, calculated in the BOX case (with $x_{b}=120$ fm, green bars) compared with analytical (dashed blue line) and "exact" results (solid red line).

\section{Two-body problem}

After having investigated different approaches to discretize the continuum in a one-body problem we proceed to a more complex, and physically more enlightening, two-body system. Our schematic model in this case consists of a fully occupied Woods-Saxon core plus two particles outside the core interacting via a matter density-dependent point contact residual interaction. As anticipated in the introduction of this memory, our goal with this choice is to model a simplified (1D) Borromean nucleus, i. e. a system with a two-particle halo which is bound notwithstanding the possible core plus one particle subsystem is unbound. This simplified 1D model has already been presented $[22,21]$ and tested in some reaction applications [7].

The two-body model will be summarized in section 3.1, the results for the system eigenvalues and eigenvectors will be shown in section 3.2, while other computed quantities of physical interest will be given in section 3.3.

\subsection{The two-body model}

The two-body problem consists of two valence particles, moving in a one-dimensional Woods-Saxon potential core (2), whose bound levels are assumed to be completely filled, interacting via a density-dependent short-range attractive residual interaction

$$
V_{\text {int }}\left(x_{1}, x_{2}\right)=V_{0} \delta\left(x_{1}-x_{2}\right)-V_{R I}\left[\frac{\rho\left[\left(x_{1}+x_{2}\right) / 2\right]}{\rho_{0}}\right]^{p} \delta\left(x_{1}-x_{2}\right),
$$


where $V_{0}, V_{R I}, p$, and $\rho_{0}$ are parameters, and $\rho(x)$ is the core matter density

$$
\rho(x)=\sum_{i=0}^{N_{b}-1} \psi_{i}^{*}(x) \psi_{i}(x)
$$

where $N_{b}$ is the number of occupied bound states. The formulation of this problem has been introduced and presented in [23] for the 3D case. Note that in our 1D reduction for a symmetric potential the core density (22) is a symmetric function of $x$. In the following, we assume that the volume term $V_{0}$ is zero and we only deal with the matter density weighted term.

Since we model a drip line system, we have chosen the Fermi surface in such a way that there are no available bound states, so the two unperturbed particles must lie in the continuum. The final two-body state becomes bound due to the action of the residual interaction between the two particles, akin to a 1D "Borromean" system. Thus, the two-body Hamiltonian $H_{2 b}$ is built combining the one-body Hamiltonian (2) with the residual interaction $(21)$

$$
H_{2 b}\left(x_{1}, x_{2}\right)=H_{1 b}\left(x_{1}\right)+H_{1 b}\left(x_{2}\right)+V_{\text {int }}\left(x_{1}, x_{2}\right) .
$$

Our strategy is to diagonalize the two-body Hamiltonian (23) in a two-body basis built with states that are above the Fermi energy surface. We proceed to detail the basis construction following [23]. The full 1D one-body wave function has two components, a spatial part and a spinor part

$$
\Psi_{n, m_{s}}^{(1 b)}(x)=\psi_{n}(x) \chi_{m_{s}}^{(s)},
$$

where the one-body spatial component has been previously obtained using any of the methods presented in section 2.1.

The two-body basis can be constructed involving products of one-body wave functions to obtain

$$
\begin{aligned}
\Psi_{n_{1}, n_{2}, S, m_{S}}^{(2 b)}\left(x_{1}, x_{2}\right)= & \psi_{n_{1}}\left(x_{1}\right) \psi_{n_{2}}\left(x_{2}\right) \\
& \times \sum_{m_{s_{1}}, m_{s_{2}}}\left\langle s_{1} m_{s_{1}} s_{2} m_{s_{2}} \mid s_{1} s_{2} S m_{S}\right\rangle \chi_{m_{s_{1}}}^{\left(s_{1}\right)} \chi_{m_{s_{2}}}^{\left(s_{2}\right)} .
\end{aligned}
$$

Assuming that we are dealing with fermions, the full wave function (25) should be antisymmetric under the interchange of the labels 1 and 2 . Thus, if we consider the singlet $S=0$ wave function, the spin degrees of freedom are antisymmetric [24]

$$
\Psi_{n_{1}, n_{2}, 0,0}^{(2 b)}\left(x_{1}, x_{2}\right)=\psi_{n_{1}}\left(x_{1}\right) \psi_{n_{2}}\left(x_{2}\right)\left[\frac{1}{\sqrt{2}}\left(\chi_{1 / 2}^{(1 / 2)} \chi_{-1 / 2}^{(1 / 2)}-\chi_{-1 / 2}^{(1 / 2)} \chi_{1 / 2}^{(1 / 2)}\right)\right] .
$$

The spatial part should be symmetrized, and the dimension of the problem for $N$ onebody spatial wave functions goes down from $N^{2}$ to $N(N+1) / 2$ for the symmetric two-body spatial wave functions $\psi_{n_{1}, n_{2}}^{(2 b)}\left(x_{1}, x_{2}\right)$

$$
\psi_{n_{1}, n_{2}}^{(2 b)}\left(x_{1}, x_{2}\right)=\frac{\sqrt{2-\delta_{n_{1}, n_{2}}}}{2}\left[\psi_{n_{1}}\left(x_{1}\right) \psi_{n_{2}}\left(x_{2}\right)+\psi_{n_{2}}\left(x_{1}\right) \psi_{n_{1}}\left(x_{2}\right)\right] .
$$


or, using the ket notation,

$$
\psi_{n_{1}, n_{2}}^{(2 b)}\left(x_{1}, x_{2}\right) \rightarrow\left|(s) n_{1} n_{2}\right\rangle=\frac{\sqrt{2-\delta_{n_{1}, n_{2}}}}{2}\left(\left|n_{1} n_{2}\right\rangle+\left|n_{2} n_{1}\right\rangle\right) .
$$

The matrix elements of the Hamiltonian (23) in the symmetrized basis are

$$
\begin{aligned}
&\left\langle(s) n_{1}^{\prime} n_{2}^{\prime}\right| H_{2 b}\left|(s) n_{1} n_{2}\right\rangle=\frac{\sqrt{\left(2-\delta_{n_{1}, n_{2}}\right)\left(2-\delta_{n_{1}^{\prime}, n_{2}^{\prime}}\right)}}{2}\left(E_{n_{1}}+E_{n_{2}}\right) \\
& \times\left(\delta_{n_{1}, n_{1}^{\prime}} \delta_{n_{2}, n_{2}^{\prime}}+\delta_{n_{1}, n_{2}^{\prime}} \delta_{n_{2}, n_{1}^{\prime}}\right)+\left\langle(s) n_{1}^{\prime} n_{2}^{\prime}\left|V_{i n t}\right|(s) n_{1} n_{2}\right\rangle,
\end{aligned}
$$

where the matrix element of the residual interaction is

$$
\begin{aligned}
& \left\langle(s) n_{1}^{\prime} n_{2}^{\prime}\left|V_{i n t}\right|(s) n_{1} n_{2}\right\rangle=-V_{R I} \sqrt{\left(2-\delta_{n_{1}^{\prime}, n_{2}^{\prime}}\right)\left(2-\delta_{n_{1}, n_{2}}\right)} \\
& \times \int_{-\infty}^{+\infty} d x \psi_{n_{1}^{\prime}}^{*}(x) \psi_{n_{2}^{\prime}}^{*}(x)\left[\frac{\rho(x)}{\rho_{0}}\right]^{p} \psi_{n_{1}}(x) \psi_{n_{2}}(x) .
\end{aligned}
$$

As the core density (22) is symmetric, the integrand has to be a symmetric function too, which implies the selection rule

$$
n_{1}+n_{2}+n_{1}^{\prime}+n_{2}^{\prime}=2 n ; \quad n=0,1,2, \ldots
$$

As we are dealing with a contact interaction, it is important to define an energy threshold, $E_{t h}$, beyond which the two-body basis components are not taken into account. Thus, only basis states $\left|(s) n_{1} n_{2}\right\rangle$ such that $E_{n_{1}}+E_{n_{2}} \leq E_{t h}$ will enter into the calculation. This energy cut-off is due to the special characteristics of the point contact interaction that forbid convergence when the full space is considered [23].

For each of the considered discretized bases (HO, THO, BOX) we solve the onebody problem (1) to obtain a set of bound states and a set of pseudostates representing the continuum. Then, using the positive-energy pseudostates, the Fermi-allowed twobody basis is built and the two-body Hamiltonian (23) is diagonalized, computing the matrix elements (29). This second part is common to all methods.

The residual interaction parameter values selected in the present work are as follows

$$
\begin{array}{lrl}
V_{0}=0.0 \mathrm{MeV}, & V_{R I}=-38.0 \mathrm{MeV}, & \rho_{0}=0.15 \mathrm{fm}^{-1}, \\
p=1, & E_{t h}=50.0 \mathrm{MeV}, & \mu=0.975 \mathrm{amu} .
\end{array}
$$

\subsection{Energies and Wavefunctions for the two-body model.}

As in the one-body case, we check the bound state energy convergence and the wave functions tails. A proper behavior in the tail region is essential in the description of twoparticle transfer processes in connection with the pairing field. We should emphasize that, in comparison with the one-body case, the treatment of this case is a more complex task, since it implies much larger bases.

In the first place we check the dependence of the resulting two-body Hamiltonian eigenvalues with the dimension of the truncated 1D basis. This is depicted in figure $8 \mathrm{a}$ for the HO and 8b THO methods, while for the BOX method the dependence on 
the box radius is reported in figure 8c. As can be seen from those figures, the energy of the bound state (red solid line) is converging, with residual minor oscillations, to a limiting value $E_{b}=-0.5 \mathrm{MeV}$. Note that without the residual interaction the two-body system is unbound, and it is the attractive residual interaction between the two valence neutrons that makes the system bound. To show the quantitative effect of the residual interaction, we also depict in figure 8, as a dashed green line, the energy of the lowest unperturbed two-particle state for each value of the parameter $\mathrm{N}$ or $x_{b}$. The dotted blue lines are the energies of the lower two-particle pseudostates in the continuum arising from the diagonalization. As in the one-body case the convergence is faster with respect to $\mathrm{N}$ for the THO case as compared to the simpler HO case.

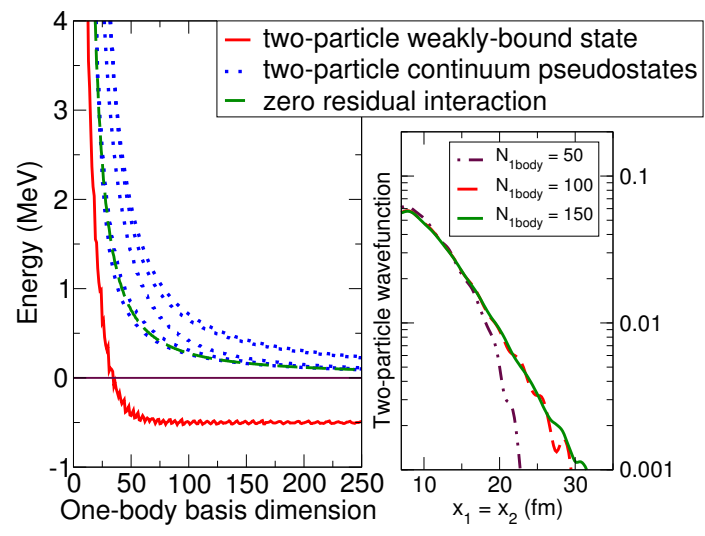

(a) $\mathrm{HO}$

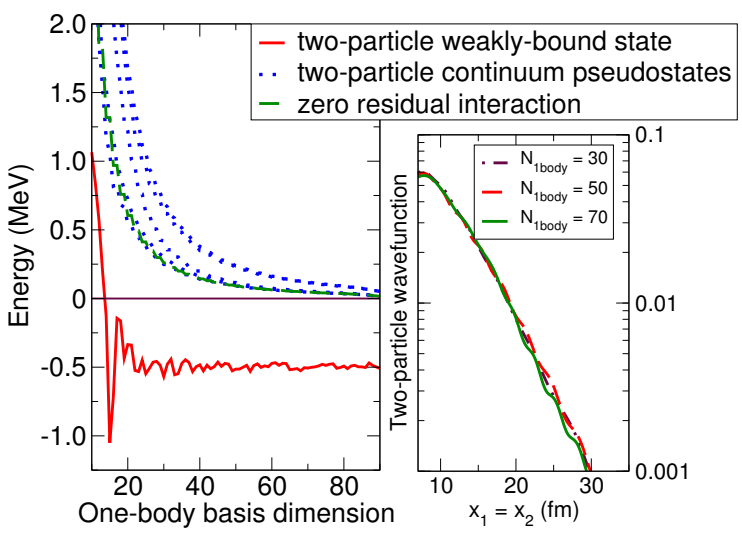

(b) $\mathrm{THO}$

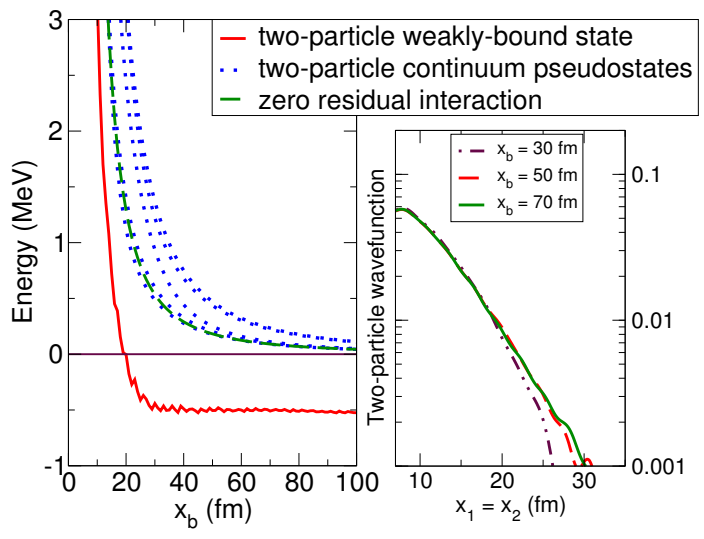

(c) BOX

Figure 8: Panel a: first two-body Hamiltonian energies (solid red and dotted blue lines) as a function of the dimension of the truncated 1D HO basis (left) and ground state wavefunction tail $\Psi(x, x)$ for different values of the one-body basis dimension (right). Panel b: same for THO basis. Panel c: first two-body Hamiltonian energies as a function of the radius $x_{b}$ of the BOX (left) and ground-state wavefunction tail $\Psi(x, x)$ for different values of the box radius (right). Also shown, as dashed green lines, the energies of the lowest unperturbed two-particle state for each value of the parameter $\mathrm{N}$ or $x_{b}$.

As in the one-body case it is important to check also the radial behavior of the 
bound eigenstate. To this end the resulting ground-state wave function $\Psi(x, x)$ for $x_{1}=x_{2}=x$ is plotted in the right panels of figure 8 for different $\mathrm{N}$ values in the HO and THO cases or $x_{b}$ for the BOX. In order to display the slow convergence in the tail region, we show in the figure just the asymptotic part of the wave functions in a logarithmic scale.

A further insight on the effect of the residual pairing correlations can be obtained by looking at the features of the wave function (or its modulus squared) as a function of the coordinates $x_{1}$ and $x_{2}$. The results obtained for the correlated two-particle ground state are displayed in the figure 9 and should be compared with the uncorrelated case displayed in figure 10. In order to better pinpoint the effect of correlations we define an uncorrelated case with zero residual interaction, but with a mean field such to obtain a two-particle uncorrelated wave function with the same total binding energy $(-0.5$ $\mathrm{MeV}$ ) as the final correlated one. It is apparent from the comparison of the two figures that the residual interaction has created a spatial correlation between the two particles, proved by an increased probability along the bisector line $x_{1}=x_{2}$, i. e. for small relative distances. On the contrary, the uncorrelated wave functions looks completely symmetric in the four quadrants, implying that the probability is not maximum for small relative distances.

The enhanced spatial correlation in the correlated case can be even better appreciated by cutting the wavefunctions presented in figures 9 and 10 along the $x_{1}=x_{2}$ bisector. Correlated and uncorrelated sections are compared in figure 11, showing the strong enhancement in the correlated case. This enhancement will be reflected an increasing probability for two-particle transfer, two-particle breakup or knock-out processes, with strong angular correlation between the two emitted particles in the two latter cases [25]. We can note that all discretization methods provide similar results, although again the number $\mathrm{N}$ of shells required to get convergence in THO is smaller than in $\mathrm{HO}$.

\subsection{Transition matrix elements for $x$ and $x^{2}$ operators}

The knowledge of the ground and excited two-particle states allows us now to calculate matrix elements of different operators, e.g. $x$ and $x^{2}$, between the ground state and all "continuum" excited states. Besides checking the different discretization methods, one can compare the energy strength distribution with those obtained within the di-neutron cluster model.

We start by extending the $x$ and $x^{2}$ operators to the two-particle case. Defining

$$
\begin{aligned}
& D_{12}=x_{1}+x_{2}, \\
& Q_{12}=x_{1}^{2}+x_{2}^{2},
\end{aligned}
$$

and we compute the transition integrals

$$
\left\langle\Psi_{b}\left|\mathcal{O}\left(x_{1}, x_{2}\right)\right| \Psi_{i}\right\rangle=\int_{-\infty}^{+\infty} d x_{1} d x_{2} \Psi_{b}^{*}\left(x_{1}, x_{2}\right) \mathcal{O}\left(x_{1}, x_{2}\right) \Psi_{i}\left(x_{1}, x_{2}\right)
$$


Bound and unbound nuclear systems at the drip lines: a one-dimensional model

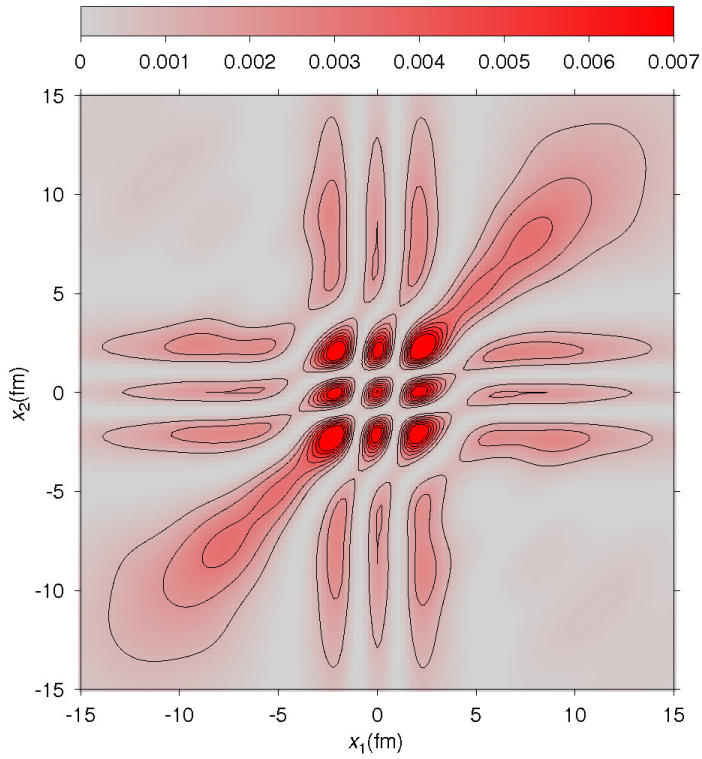

(a) $\mathrm{HO}$

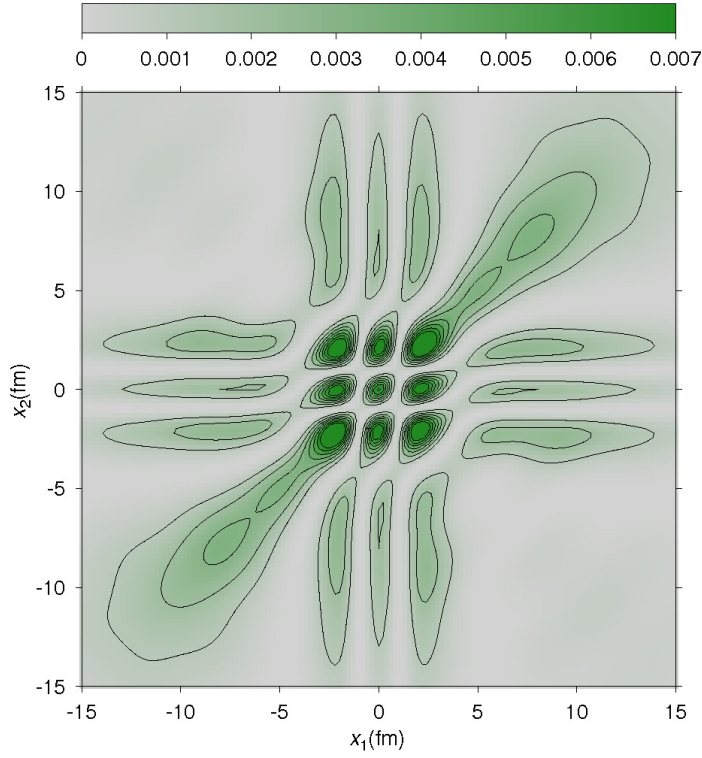

(b) $\mathrm{THO}$

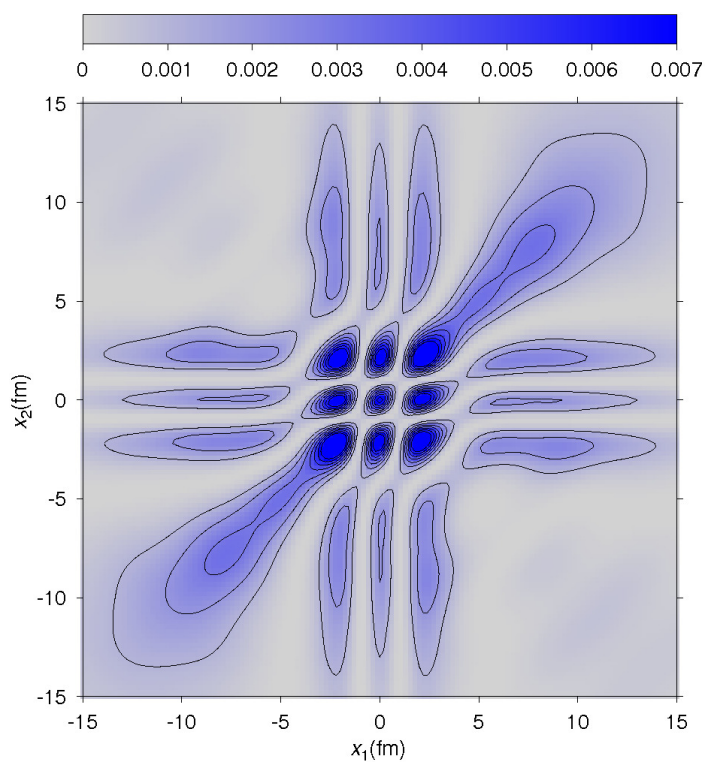

(c) BOX

Figure 9: Contour plots of the probability density for the two-body bound state with binding energy $E=-0.5 \mathrm{MeV}$ using the correlated Hamiltonian (23) constructed with different bases: HO with $N=200$ in panel (a), THO with $\gamma / b=1.2 \mathrm{fm}^{-1 / 2}$ and $N=75$ in panel (b), and a BOX with $x_{b}=100 \mathrm{fm}$ in panel (c). 


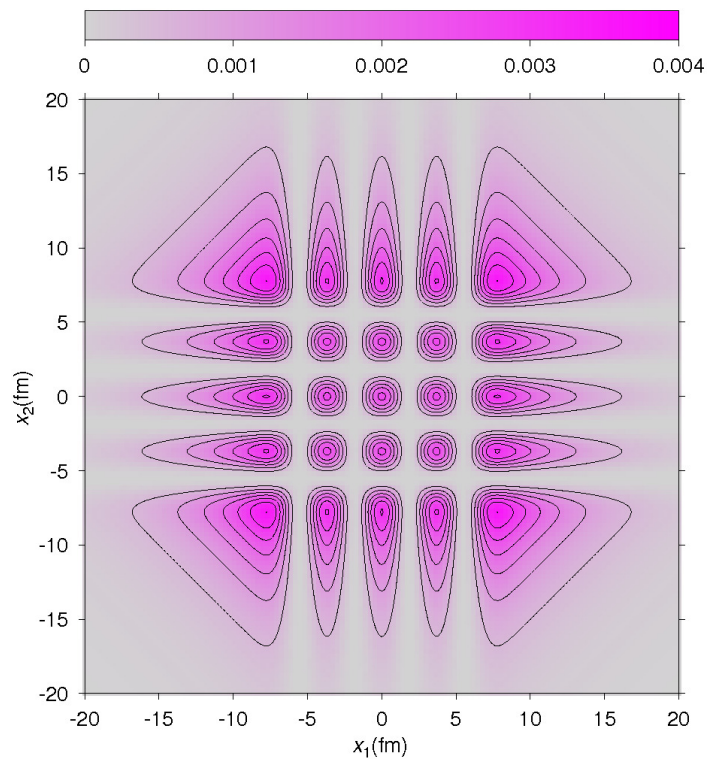

Figure 10: Same than figure 9, but for the wave function obtained with an uncorrelated Hamiltonian (zero residual interaction), with a single particle potential depth modified to obtain for the two-particle system the same binding energy as in the correlated case $(E=-0.5 \mathrm{MeV})$.

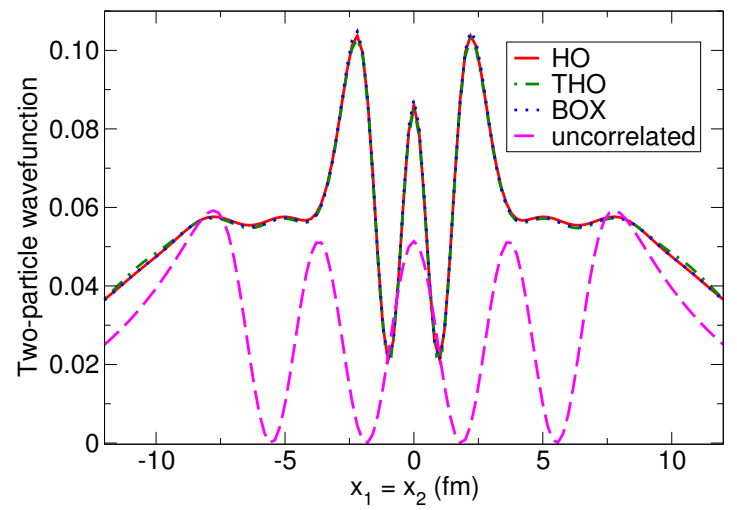

Figure 11: Two-body bound state along the $x_{1}=x_{2}$ bisector for the correlated and uncorrelated cases presented in figures 9 and 10 .

where $\mathcal{O}\left(x_{1}, x_{2}\right)=D_{12}$ or $Q_{12}, \Psi_{b}\left(x_{1}, x_{2}\right)$ is the two-body ground state, and the set $\left\{\left|\Psi_{i}\left(x_{1}, x_{2}\right)\right\rangle\right\}$ reptesents the two-body continuum pseudostates.

The resulting distribution of modulus squared matrix elements from the ground state to continuum states is reported in figure 12 for the different discretization methods and for different values of $\mathrm{N}(\mathrm{HO}$ and $\mathrm{THO})$ or $x_{b}(\mathrm{BOX})$. Upper frames refer to $B_{1}=$ $\left|\left\langle\Psi_{b}\left|D_{12}\right| \Psi_{i}\right\rangle\right|^{2}$, lower frames to $B_{2}=\left|\left\langle\Psi_{b}\left|Q_{12}\right| \Psi_{i}\right\rangle\right|^{2}$. Since the different calculations lead to different densities of states, for a better comparison of the convergence properties we 
also display in the legends of the figure the integrated values.

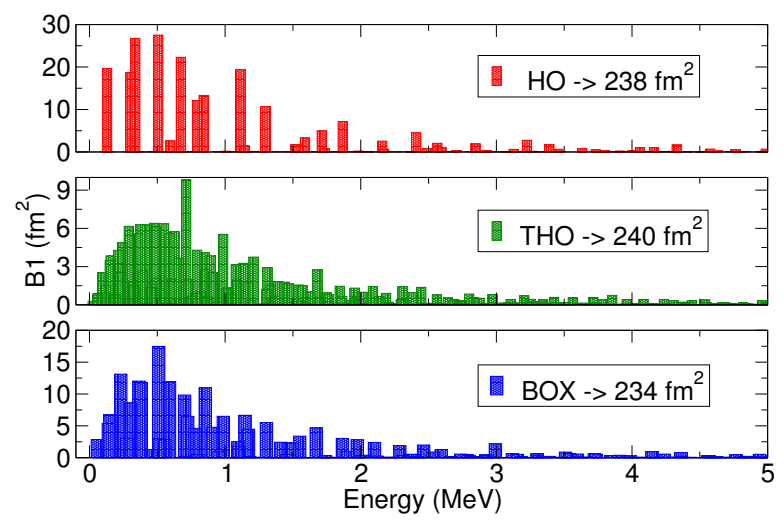

(a) B1

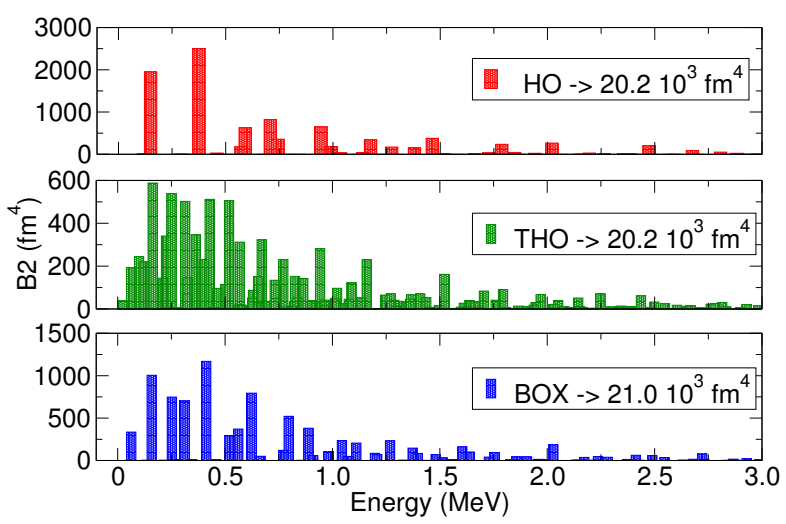

(b) B2

Figure 12: Results for the $\mathrm{B}_{1}$ and $\mathrm{B}_{2}$ transition intensities in the microscopic twobody approach as a function of the excitation energy, using the different discretization methods. Numbers in each panel legend are the integrated values. In the HO case the basis dimension is $\mathrm{N}=200$, in the $\mathrm{THO}$ case $\mathrm{N}=100$, and for the BOX case the radius is $x_{b}=90 \mathrm{fm}$.

It would be natural trying to interprete the above results in terms of sum rules. This is more complex in the case of the TSSR see (13), since it would require the evaluation of the expectation value of $x_{1} x_{2}$ operator. It is, on the other hand, easier to evaluate the EWSR: in an A-body system, for one-body operators, it can be calculated as

$$
\mathcal{E}_{W}^{(b)}\left(x_{1}, \ldots, x_{A}\right)=\frac{1}{2} \frac{\hbar^{2}}{\mu}\left\langle\Psi_{b}\left|\sum_{i=1}^{A}\left(\frac{\partial \mathcal{O}\left(x_{i}\right)}{\partial x_{i}}\right)^{2}\right| \Psi_{b}\right\rangle .
$$

The fulfillment of the EWSR is an indication of the goodness of the discretization method, but also of the completeness of the basis, as stated for the one-body case in Section 2.3. The EWSR value is in fact only recovered when a complete basis is used which is not our case. Since we are dealing with a delta function, we truncate all bases up to a certain energy. Since the values of the transition matrix elements are rapidly decreasing (cf. figure 12) we may assume that the cut-off is not so relevant. However, 
it is much more important that in our model we have assumed a saturated and frozen core: states that correspond to both particles in a "forbidden" bound state or states which represent combinations of one particle in a core state and another in a one-body continuum state have not been included in the two-body basis. For this reason the comparison with the EWSR for the two-body case is beyond our simple approach.

We conclude this section by comparing the results obtained in the two-body model with the corresponding distribution of matrix elements obtained within the di-neutron cluster model [26]. To this end we have considered a $1 \mathrm{D}$ cluster of two neutrons with mass $\mu=1.885$ amu moving in a potential defined in such a way to reproduce the same binding energy $(\mathrm{E}=-0.50 \mathrm{MeV})$ and quantum numbers of the two-body model case:

$$
V_{0}=-50.9 \mathrm{MeV}, \quad R=5.0 \mathrm{fm}, \quad \alpha=0.4 \mathrm{fm} .
$$

In this case the transition intensities to continuum are calculated with a one-body operator $x_{\text {clus }}$, the c.m. coordinate of the cluster, and the basis states are single-particle bound and pseudo states $\left\{\left|\Phi_{i}\right\rangle\right\}$ generated by the "cluster" potential. In lower panel of figure 13 we present the cluster response $B_{1}=\left|\left\langle\Phi_{b}\left|x_{c l u s}\right| \Phi_{i}\right\rangle\right|^{2}$ with the corresponding two-body calculations $B_{1}=\left|\left\langle\Psi_{b}\left|x_{1}+x_{2}\right| \Psi_{i}\right\rangle\right|^{2}$. For this comparison we have chosen THO results with $\mathrm{N}=100$ for the "microscopic" case and $\mathrm{N}=200$ for the cluster case. The two distributions have the same shape but, apart from a different normalization factor, the maxima are not located at the same energy. This suggests that the position of the peak in the $\mathrm{B}(\mathrm{E} 1)$ distribution could provide a hint on the possible validity of the popularly used "di-neutron cluster" model.

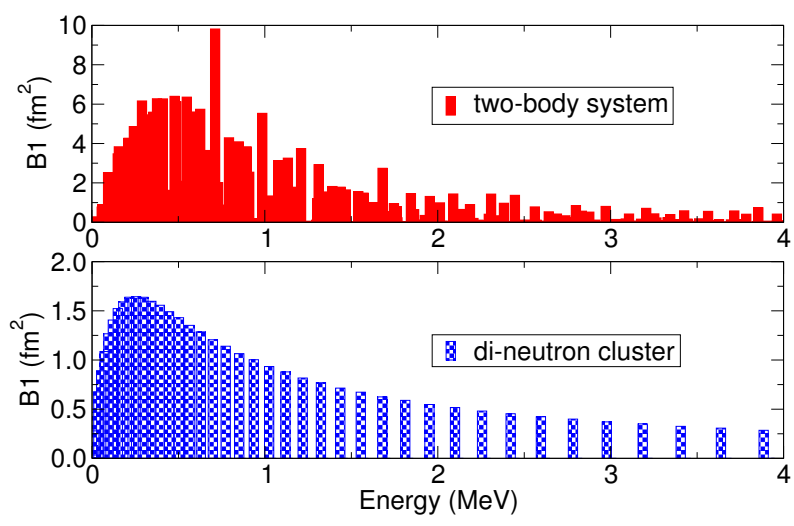

Figure 13: Results for the $\mathrm{B}_{1}$ transition intensities in the microscopic two-body approach (upper panel, $\mathrm{THO}$ with $\mathrm{N}=100$ ) compared with the di-neutron cluster calculation (lower panel, THO with $\mathrm{N}=200$ ).

\section{Summary and Conclusions}

The goal of the present memory is to investigate aspects of a weakly-bound 1D quantum two-particle system. The 1D simplified model permits a clear description of the relevant 
physical parameters without the mathematical complexities associated with the full 3D case.

The model system is composed of a core that is a mean field potential modeled by a Woods-Saxon, plus two valence nucleons bound by a matter density-dependent delta residual interaction. The inclusion of the continuum in the description of the chosen system is absolutely mandatory and we have explored three different discretization (HO, THO, and BOX). We have obtained the bound state and pseudostates (positive energy states) of the system. Pseudostates are considered a finite and discrete representation of the two-body continuum spectrum. Note that in order to construct the basis for the two-body case we need the solution of the one-body mean field potential, both for bound states and pseudostates.

In the one-body case, in addition to the calculation of the system eigenstates we compute transition intensities and sum rules of different operators to assess the goodness of the continuum description achieved with the different methods. Besides, we compare our results with "exact" and approximate approaches, thus probing the validity of the different discretization procedures.

In the two-body case, we build the two-particle bases, compute the system eigenvalues and eigenvectors, and study the bound state energy convergence and the nature of its wave function. We also compute the transition intensities for the two-body system for the linear $\left(\mathrm{B}_{1}\right)$ and quadratic $\left(\mathrm{B}_{2}\right)$ operators. We compare our $\mathrm{B}_{1}$ result with the dineutron approximation result, showing how the distributions peak at different energies. We suggest that this fact could be used to discriminate among the two approaches.

As already mentioned in the introduction, we have developed the computer codes required to perform the calculations included in the memory [8].

The results obtained in the one- and two-body cases for HO, THO, and BOX agree satisfactorily. The pseudostate method turns out to be a computationally efficient approach to deal with weakly-bound systems.

In the one-body case the system is so simple that large bases (several hundred elements) can be used requiring a very small computing time. However, for the two-body system, despite the symmetrization and energy threshold, the involved matrices dimension is much larger and the problem is computationally heavier. In fact, this is the main reason to support the use of a THO basis, that requires smaller dimension. Despite the necessity of calculating an optimal $\gamma / b$ ratio for the problem under study, the THO basis offers two important advantages. The first is the possibility of tuning the density of continuum states, making possible to enlarge the pseudostates density at energies relevant for the process under study. The second advantage is the exponential asymptotic behavior of its basis elements. The combination of these two facts makes the convergence in the THO case faster than in the other two methods. Though in the present case this advantage is not decisive, in more involved many-body calculations it can be of major relevance. 
The present results open up many possible lines to proceed the investigation on weakly-bound systems. We list some promising research lines for the future:

- the simplicity of the present approach allows its use to model transfer reactions and breakup processes in a simplified and schematic way, along the line already displayed in [25, 27, 28];

- the present model could provide a convenient way of reckoning the importance of the pairing interaction in the continuum and the effect of resonances [29] though the absence of centrifugal barrier in our 1D model implies in the latter case the introduction of an ad-hoc barrier in the potential;

- the inclusion of core excitations in the model opens up also a very enticing line of research. These core excitations have been proved to play an important role in the determination of the structure of some halo nuclei $[30,31]$ and this model could offer a convenient (and simple) test ground for their study.

\section{Acknowledgments}

The authors wish to thank José M. Arias, José E. García-Ramos, and Antonio Moro for useful discussions. Part of this work was funded by MINECO grant FIS2014-53448C2-2-P. LM thanks the University of Huelva, where part of this work was done with the finantial support of the Erasmus Placement program.

\section{Appendix A: a procedure for the HO inverse oscillator length optimization}

In accordance with other cases the value of this parameter has been fixed to minimize the ground state energy with a small HO basis [15]. In fact, we could use an $N=1$ basis, that is, a basis with the $\mathrm{HO}$ ground state as its only component, to obtain a crude approximation to the system ground state energy. In this way $a$ is a variational parameter. However, in cases like the model Woods-Saxon potential, with a weaklybound state in addition to other bound states, the $a$ value obtained using only the ground state is too large, and the resulting harmonic potential too narrow. This implies that it is necessary a very large HO basis dimension to sample the large spatial region where the tails of the weakly-bound state are still significant. To overcome this problem one should use a smaller $a$ value. This can be done manually, though we have deviced the following algorithm. Let us assume that, as in the selected study case, the potential has three bound states and the last one is weakly bound. We first make the minimization explained above; then with the obtained $a$ value we build the basis and the system Hamiltonian is diagonalized. At this point it is possible to evaluate the expectation value of the $x^{2}$ operator for the weakly bound wave function i. e. $\left\langle\Psi_{2}^{H O}\left|x^{2}\right| \Psi_{2}^{H O}\right\rangle$. We then compare this result with the same matrix element calculated for a $\mathrm{HO}$ basis. We can set a

new inverse oscillator length $a$ equating $\left\langle\Phi_{2}^{H O}\left|x^{2}\right| \Phi_{2}^{H O}\right\rangle=\frac{5}{2} a^{-2}$ to $\left\langle\Psi_{2}^{H O}\left|x^{2}\right| \Psi_{2}^{H O}\right\rangle$. Once the new parameter is obtained, we reconstruct the basis and diagonalize again before 
proceeding to the observable calculation. The optimization of the inverse oscillator length allows to construct a $\mathrm{HO}$ basis which encompasses the full bound states spatial range.

\section{References}

[1] Tanihata I 1996 J. Phys. G: Nucl. Part. Phys. 22157

[2] Tanihata I, Savajols H and Kanungo R 2013 Prog. Part. Nucl. Phys. 68 215-313

[3] Jensen AS, Riisager K, Fedorov DV and Garrido E 2004 Rev. Mod. Phys. 76215

[4] Bertulani CA and Bertsch GF 1993 Nucl. Phys. A 556 136-46

[5] Bertulani CA and Bertsch GF 1994 Phys. Rev. C 492834

[6] Dasso CH and Vitturi A 2009 Phys. Rev. C 79064620

[7] Hagino K, Vitturi A, Pérez-Bernal F and Sagawa H 2011 J. Phys. G: Nucl. Part. Phys. 38015105

[8] We have also made use of NAG, ATLAS-LAPACK, and LAPACK95 libraries. In these programs the following values of the relevant physical constants are used: $\hbar c=197.32858 \mathrm{MeV} \mathrm{fm}$, $a m u=938.92635 \mathrm{MeV} / \mathrm{c}^{2}$ and $\hbar^{2} / a m u=41.4713768 \mathrm{MeV} \mathrm{fm}{ }^{2}$. The code is available under request.

[9] L Moschini, Description of bound and unbound many-body systems at the drip-lines, Master's Thesis, Università degli Studi di Padova, 2013 arXiv:1410.7167

[10] Id Betan R, Dussel GG and Liotta RJ 2008 Phys. Rev. C 78044325

[11] Hazi AU and Taylor HS 1970 Phys. Rev. A 1, 4, 1109-20

[12] Abramowitz M and Stegun IA, Handbook of Mathematical Functions, Dover Publications, 1965

[13] Pérez-Bernal F, Martel I, Arias JM and Gómez-Camacho J 2003 Phys. Rev. A 67052108

[14] Moro AM, Arias JM, Gómez-Camacho J and Pérez-Bernal F 2009 Phys. Rev. C 80054605

[15] Lay JA, Moro AM, Arias JM and Gómez-Camacho J 2010 Phys. Rev. C 82024605

[16] Karataglidis S, Amos K and Giraud BG 2005 Phys. Rev. C 71064601

[17] Lay Valera JA, Descripción de la estructura y reacciones de núcleos exóticos en una base de oscilador armónico transformado, PhD Thesis, Universidad de Sevilla, 2012

[18] Lipparini E and Stringari S 1989 Phys. Rep. 175 103-261

[19] Bohigas O, Lane AM and Martorell J 1979 Phys. Rep. 51 267-316

[20] Moyer CA 2006 Comp. Sci. Eng. 8 32-40

[21] Vitturi A and Pérez-Bernal F 2012 AIP Conference Proceedings 1488 220-26

[22] Vitturi A and Pérez-Bernal F 2010 Nucl. Phys. A $\mathbf{8 3 4}$ 482c

[23] Bertsch GF and Esbensen H 1991 Ann. Phys. 209327

[24] The triplet contribution of the spin can be considered in connection with an antisymmetrized spatial part, but in this case the interaction should not be of a contact type and $x_{1} \neq x_{2}$.

[25] Vitturi A, Moschini L, Hagino K and Moro A 2015 AIP Conference Proceedings 1681060001

[26] Moro A, Rusek K, Arias JM, Gómez-Camacho J and Rodriguez-Gallardo M 2007 Phys. Rev. C 75064607

[27] Moschini L 2014 J. Phys: Conf. Ser. 566012027

[28] Vitturi A and Moschini L 2015 J. Phys: Conf. Ser. 590012007

[29] Id Betan R, Dussel GG and Liotta RJ 2008 Phys. Rev. C 78044325

[30] Kato K, Yamada T and Ikeda K 1999 Prog. Theor. Phys. 101 119-38

[31] Ikeda K, Myo T, Kato K and Toki H 2010 Clusters in Nuclei, Lecture Notes in Physics 818 $165-221$ 